\title{
BMJ Open Self-reported physical and mental health of Australian carers: a cross-sectional study
}

\author{
Rafat Hussain, ${ }^{1}$ Stuart Wark, ${ }^{2}$ Gina Dillon, ${ }^{2}$ Peta Ryan ${ }^{2}$
}

To cite: Hussain R, Wark S, Dillon $\mathrm{G}$, et al. Self-reported physical and mental health of Australian carers:

a cross-sectional study. BMJ Open 2016;6:e11417. doi:10.1136/bmjopen-2016011417

- Prepublication history and additional material is available. To view please visit the journal (http://dx.doi.org/ 10.1136/bmjopen-2016011417).

Received 8 February 2016 Revised 1 August 2016 Accepted 17 August 2016

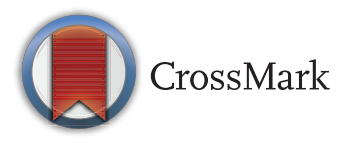

${ }^{1}$ ANU School of Medicine \& Research School of Population Health, Australian National University, Canberra, Australian Capital Territory, Australia

${ }^{2}$ School of Rural Medicine, University of New England, Armidale, New South Wales, Australia

Correspondence to Dr Rafat Hussain; Rafat.Hussain@anu.edu.au

\section{ABSTRACT}

Objective: To report on self-reported physical and mental health of informal carers in rural regions of New South Wales, Australia.

Methods: A cross-sectional community-based sample $(n=222)$ of carers completed a questionnaire incorporating self-reported measures of health from validated international instruments including Medical Outcomes Study Scale (SF-36), the Centre for Epidemiology-Depression (CES-D) and Kessler-10 (K-10) Psychological Distress Scales, along with information on participant demographics and other key caregiving characteristics such as health condition of care recipient.

Results: Rural carers' self-reported health was poor as evident on the SF-36 Physical and Mental Health component scores as well as each individual domain of the SF-36. Results from the CES-D and K-10 scores indicated very high rates of depressive symptoms and psychological distress. Over $70 \%$ of carers within the current study had CES-D scores indicative of depressive symptoms. Scores on the K-10 indicate almost half the carers were experiencing high levels of psychological distress, which is over 4 times the rate reported in the general Australian population.

Conclusions and implications: Results from this study were compared to Australian population normative data and were found to be significantly below Australian age-matched population norms for SF-36, CES-D and K-10. These findings illustrate the poor health profile of informal carers relative to the general Australian population, especially in terms of depressive symptoms and psychological distress. This highlights the need for additional support for rural carers in order to ease the accumulated mental and physical health burdens of this group.

\section{INTRODUCTION}

The Australian Government has a stated commitment to supporting individuals with various health needs, such as a disability, ageing-related impairment and physical or mental health issue, to be assisted to remain within their local community. ${ }^{1}{ }^{2}$ However, the support provided by the government is

\section{Strengths and limitations of this study}

- This study specifically focuses on the selfreported health of carers in non-metropolitan Australia.

- It was found that the health of rurally located carers was significantly below Australian agematched population norms, with over $70 \%$ of carers showing depressive symptomology.

- Almost half the carers were found to be experiencing high levels of psychological distress.

- This study methodology contains the risk of selfselection bias and problems associated in the self-reporting of health conditions.

- There was a significant gender bias towards female respondents, so any sex-based comparisons must be viewed with caution.

not always comprehensive and often requires significant assistance from informal carers. Informal care is a support model where a person provides unpaid assistance to another individual with respect to general activities of daily living such as physical, emotional, financial and personal care. ${ }^{3}$ The support of the informal carer is often vital, playing a significant role in increasing the individual's access to health and rehabilitation services. ${ }^{4}$

The 'burden' of providing informal care is considered to be the reduction in personal opportunities or actual health of an individual as a direct result of having to provide this unpaid support. ${ }^{5}$ It has been previously identified that informal carers are likely to experience high levels of psychological distress ${ }^{6}$ and a decline in physical health ${ }^{7}$ and quality of life. ${ }^{8}$ The need to provide informal care, particularly personal care, ${ }^{9}$ has an impact on the carer's ability to seek and maintain external paid employment. ${ }^{10}$ It was estimated in 2015 that 2.86 million people in Australia provide informal care support to another person. ${ }^{11}$ This figure represents $\sim 12.5 \%$ of the nation's population and an increase of around 260000 carers from 2012. ${ }^{12}$ However, it is concerning to note that there are now 
perceived to be fewer carers, relative to demand, than in 2010, and the demand for informal care is predicted to considerably exceed its supply within the next decade. ${ }^{11}$

Although there are a number of Australian studies on caregiving, many have either looked at specific health issues such as chronic heart conditions, ${ }^{13}$ motor neuron and other similar degenerative diseases, ${ }^{14}{ }^{15}$ Alzheimer's or dementia, ${ }^{16-18}$ specific cancers ${ }^{19}$ and palliative care and/or end-of-life care issues. ${ }^{20}{ }^{21}$ Almost all studies on carers' health with the exception of a recent qualitative study ${ }^{22}$ have been conducted in large cities and metropolitan areas. It is well established that people living in rural regions in Australia face significant challenges in relation to health and social services, and this background merits specific focused investigations. ${ }^{23}$

In rural areas of Australia, the issues faced by informal carers may be amplified compared to those in metropolitan locations. ${ }^{24}$ Rural carers are often geographically isolated and struggle to access relevant healthcare support services for themselves and the person for whom they provide care. ${ }^{25}{ }^{26}$ A failure to receive appropriate and timely treatment magnifies the issues for carers, as the existing health condition then worsens progressively over time ${ }^{27}$ which then places additional stress onto the carer. ${ }^{28}$ Individuals in rural areas are less likely to receive formal carer training to cope with these concerns, ${ }^{29}$ and this in turn can lead to issues with poor self-esteem. ${ }^{30}$

In spite of these issues, there remains only limited research that specifically examines the issues and caregiver burden facing individuals in rural areas of Australia. ${ }^{15}{ }^{31}$ The current paper is part of a larger study focusing on the experiences of informal carers in nonmetropolitan areas of New South Wales (NSW), Australia. The aim of this research is to establish an initial profile of self-reported physical and mental health of rurally based informal carers using validated international health scales. A secondary aim is to assess these self-reported measures against Australian normative data and facilitate future comparative studies with metropolitan-based peers. Formal ethical approval for this project was granted by the University of New England's Human Research Ethics Committee (HREC approval number HE13/130).

\section{METHODS}

\section{Study design, setting and participants}

A cross-sectional community-based survey was undertaken in rural areas of NSW. NSW is the most populous state in Australia (total 23 million) comprising 7.1 million individuals of which nearly $20 \%$ live in rural and regional areas. ${ }^{32}$ The rural or non-metropolitan cohort of the population, as defined by the Australian Standard Geographic Classification (ASGC) system, is dispersed over a large geographic footprint comprising populations resident in very diverse regional towns and a small proportion in rural and very remote regions. ${ }^{32} 33$ The
ASGC is a nationally standardised measure of geographic remoteness, which incorporates aspects of distance and access to services in order to define five remoteness categories, namely major cities, inner regional, outer regional, remote and very remote areas. $^{34}$

Potential participants for this project were defined as being any individual over the age of 18 who selfidentifies as providing informal care and support for a person with either a disability or other long-term health condition. The geographic catchment was specified as being areas of NSW that are outside of the ASGC remoteness category of 'major cities'. In order to overcome the challenge associated with the wide geographical dispersal of carers, a survey tool was developed. A cross-sectional survey questionnaire was designed and piloted in print and online format with 10 people, with expert knowledge of rural health and carer health issues. Minor modifications were made to some of the items in the survey form based on feedback from the pilot study.

A variety of community-based recruitment strategies were used to distribute information about the project and enrol participants. These included social media, community-group and carer organisation newsletters, posters in community facilities and a combination of electronic and traditional media sources such as radio and newspaper interviews. A US $\$ 5$ grocery voucher incentive was provided for all respondents. Potential participants were given the option of receiving a hard copy of the information package and associated questionnaire or to access the survey via a purpose-developed website that contained all relevant documentation and an online version of the survey questionnaire.

A total of 237 participants completed the survey of which 222 were considered valid responses from rural areas of NSW. The majority of the surveys (nearly 90\%) across the state were completed online. As most of the survey responses were online and recruitment was undertaken through a variety of community-based strategies, an overall response rate could not be determined. Participation was voluntary and anonymous. This also meant that there was no capacity to refer individuals potentially at risk for support, however, the information sheet outlining details of the study provided to all potential participants preceded the beginning of the survey questionnaire and included specific details to facilitate access to free counselling support if required through Lifeline Telephone Support Service, and the relevant toll-free telephone number was provided. Lifeline counsellors do suggest callers to contact their preferred doctor or a psychologist if symptoms have been ongoing. This strategy was approved by the Human Research Ethics Committee as a viable option to maintain anonymity of participants while also providing information on how to access counselling support for those experiencing distress associated with their caregiving role. 


\section{Data measurement}

The outcome variables included self-reported health using three validated scales: the Medical Outcomes Short-Form (SF-36), which measures health across eight domains of physical and mental health; the Centre for Epidemiologic Studies-Depression Scale (CES-D) and the Kessler-10 (K-10) Psychological Distress Scale. Each of these scales is briefly discussed below.

The SF-36 comprises of 36 questions that focus on general health, physical functioning, role physical, bodily pain, vitality, social functioning, mental health and role emotional. These eight subscales are also used to calculate two summary or component scales: the Physical Component Score (PCS), reporting on physical health, and the Mental Component Score (MCS), reporting on mental health. ${ }^{35}$ Scores on each of the SF-36 domain are standardised, and component scores can range from 0 to 100 and higher scores indicate better health. The SF-36 imputation was undertaken by Clinical Research Design IT and Statistical Support (CReDITSS), which is the statistical consulting arm of the Centre for Clinical Epidemiology and Biostatistics (CCEB) at the Hunter Medical Research Institute affiliated with University of Newcastle, Australia. CCEB have an established algorithm for missing values, which has been used by numerous research projects across Australia. Imputation of missing values and computation of the domain and component scores were performed according to the procedures outlined in the SF-36 Manual and Interpretation Guide. ${ }^{35}$ The SF-36 domain generation was carried out using the SAS software. The SAS codes contained in the document http://www.sascommunity.org/ sugi/SUGI94/Sugi-94-168\%20Newvine.pdf was used to create the nine domains (eight original and the summary component scores for PCS and MCS) using the SF36 variables. For missing data imputation, it was assumed missing data were completely at random and used person-mean imputation for individuals with missing values. Missing values on an item were replaced with the mean of all the individual's completed items. Person-mean imputation has been shown to be reliable when the numbers of respondents with missing items are $20 \%$ or less. ${ }^{36}$ The imputed data variables were then exported and reinserted in the SPSS data file before undertaking analyses for the present paper. Australian population normative data for SF-36 scores were accessed from the Australian Bureau of Statistics. ${ }^{37}$

Self-reported mental health was additionally assessed via the CES-D Scale and K-10 Psychological Distress Scale. The CES-D is a validated and widely used scale designed to measure current levels of depressive symptoms within the general population. ${ }^{38}$ CES-D scores have a possible range of $0-60$, and higher scores indicate worse mental health. A score of 16 points or more is generally accepted as an indicator of depression symptoms, ${ }^{39}$ and scores of 24 and over are suggestive of severe depressive symptoms. ${ }^{40}$ Normative CES-D data for the Australian population were derived by entering the cut points for depressive and severe depressive symptoms (16 and 24, respectively) into the MoodScore computer programme of Crawford et $a l,{ }^{41}$ which has been developed as a means of quickly referencing Australian normative data on a range of self-reported mood scales.

The K-10 Scale provides a measure of non-specific psychological distress based on questions about negative emotional states experienced in the past 4-week period. ${ }^{24}$ Scores on the K-10 range from 10 to 50 with higher scores indicating higher levels of psychological distress. The designated cut-off scores for low (10-15), moderate (16-21), high (22-29) and very high (30-50) levels of psychological distress. Australian population norms were drawn from Australian Bureau of Statistics data. $^{42}$

Information was collected on a range of explanatory variables including sociodemographic and caring role characteristics of participants including sex, age, employment status, whether they are in a primary caregiving role, their relationship to the person they provide care for (spouse/parent/child/other), whether they are aware of, or members of, any carer support groups, whether they are a live-in carer and the medical condition/s of the care recipient. The medical conditions were classified into four broad categories of

- cognitive: this includes autism spectrum disorders, Alzheimer's disease and other dementias, intellectual disability, Down syndrome and acquired brain injury;

- physical: this includes cancer, stroke, postoperative recovery, Parkinson's disease, cerebral palsy, quadriplegia, blind, deaf, diabetes, heart and lung conditions and frail ageing;

- mental health: this includes schizophrenia, attention deficit hyperactivity disorder, bipolar disorder, alcohol and drug addiction, depression and anxiety disorders;

- multiple: this category involves the coexistence of conditions across more than one of the cognitive, physical or mental health categories.

Issues relating to potential bias including selection and measurement bias are discussed in the Limitations section.

\section{Statistical methods}

Analysis was performed using SPSS V.22 (2013 release) for univariate and multivariable analyses. ANOVA with post hoc comparison via Dunnett's $\mathrm{C}$, with $\mathrm{p} \leq 0.05$ as the critical value, was used to test for differences between mean SF-36 scores of participants. Dunnett's G was recommended for use with SF-36 data as it provides very tight type 1 error control and performs well when the group sizes are different, when population variances are different or when data are not normally distributed. ${ }^{43}$ Graphical representation and comparison of SE error bars were used to gauge significant difference between mean SF-36 scores of participants from the current study and Australian population norms. In order to age-match data from the current study with ABS data for the SF-36 
population norms, the average age of participants was calculated for each age grouping in the current study. This average age was then compared to the ABS data age categories, and the normative data for the relevant category were used as a comparison to the current study.

A multiple linear regression technique was used for multivariable analyses for two of the four dependent variables, namely-PCS and MCS to estimate the proportion of variance in PCS and MCS scores that could be accounted for by age, gender, employment status, type of condition that care is being provided for and membership of a carer support group. Key assumptions were evaluated prior to interpreting the results of the multiple linear regression analyses. A visual inspection of the normal probability plot of standardised residuals and of the scatterplot of standardised residuals against standardised predicted values was undertaken. These indicated that assumptions of normality, linearity and homoscedasticity of residuals were met. Multivariate outliers were not of concern; for all cases in the data file the Cook's distance value was $<1$ and Mahalanobis distance did not exceed the critical $\chi^{2}$ for $\mathrm{df}=8$ (at $\alpha=0.001$ ) of 26.125 .

Logistic regression analysis was used individually for the remaining two dependent variables-CES-D and $\mathrm{K}-10$ using enter technique for inclusion of explanatory variables ( ables included in both regression models were age (categorical), gender, work status, type of condition that care is being provided for and membership of a carer support group.

\section{RESULTS}

The findings are based on responses from 222 participants from rural NSW. The demographic characteristics of the study sample are summarised in table 1. Raw percentages rather than cumulative percentages are reported; as there are missing values the final totals do not necessarily tally to $100 \%$. The majority of participants were women $(85 \%)$, live-in carers $(75 \%)$, involved in the primary care role $(80 \%)$. Ages ranged from 21 to 86 years, with more participants being from the older age groups. Approximately $45 \%$ of the carers were also working in paid employment. Just over one-third of the participants (38\%) were caring for someone with a physical condition, and $27 \%$ of carers were caring for people with multiple conditions.

\section{Self-reported health}

Carers in the study had self-reported physical and mental health that was significantly below Australian agematched population norms. This was evident on the SF-36 Physical Component Scores (PCS) and Mental Component Scores (MCS), as well as within each individual domain of the SF-36 (figure 1). Carers showed particularly lower comparative scores in the domains of social functioning, role emotional, role physical and vitality. The comparison between findings of the present
Table 1 Demographic and caregiving characteristics of participants $(n=222)$

\begin{tabular}{lc}
\hline Participant characteristics & $\mathbf{n}(\%)$ \\
\hline Sex & $191(84.9)$ \\
Female & $31(13.8)$ \\
Male & \\
Age (years) & $21-86$ \\
$\quad$ Range & 52.7 \\
Mean & 14.4 \\
SD & \\
Age (grouped-years) & $47(21.2)$ \\
40 and under & $53(23.9)$ \\
$41-50$ & $58(25.8)$ \\
$51-60$ & $64(28.4)$ \\
61+ & \\
Currently working & $98(45.2)$ \\
Yes & $119(54.8)$ \\
No & $180(80.0)$ \\
Caregiver role & $19(8.4)$ \\
Primary & \\
Secondary & $27(12.0)$ \\
Caring relationship & $92(40.9)$ \\
Caring for a parent & $77(34.2)$ \\
Caring for a child & $17(7.6)$ \\
Caring for spouse & \\
Other & $142(75.5)$ \\
Live-in carer & $46(24.4)$ \\
Yes & \\
No & $81(37.7)$ \\
Condition cared for & $48(22.3)$ \\
Physical & $27(12.6)$ \\
Cognitive & $59(27.4)$ \\
Mental health & \\
Multiple categories & $152(67.6)$ \\
Aware of any carer support groups & \\
Yes & $61(27.1)$ \\
Member of a carer support group & \\
Yes & \\
\hline & \\
\hline &
\end{tabular}

study and the Australian normative data for summary measures of SF-36, namely, PCS and MCS as well as the scores for each of the eight individual domains, is outlined in the 'Discussion' section.

Results from the CES-D and K-10 Scales indicate very high rates of depressive symptoms and psychological distress among the rural carers sample (figure 2 and online supplementary figure S1). Over $70 \%$ of carers within the current study had results on the CES-D that indicated the presence of depressive symptoms, with $36 \%$ meeting the criteria for severe depressive symptoms. Scores on the K-10 indicate that almost half the carers $(49.3 \%)$ were experiencing high and very high levels of psychological distress. Comparison with normative data for SF-36 in Australian population is provided in the 'Discussion' section.

Factors associated with self-reported health in rural carers In table 2, we present the bivariate results for SF-36 summary scores as well as the eight individual domains 
Figure 1 Mean SF-36 scores for rural carers compared to Australian population norms. MCS, Mental Component Score; PCS, Physical Component Score.

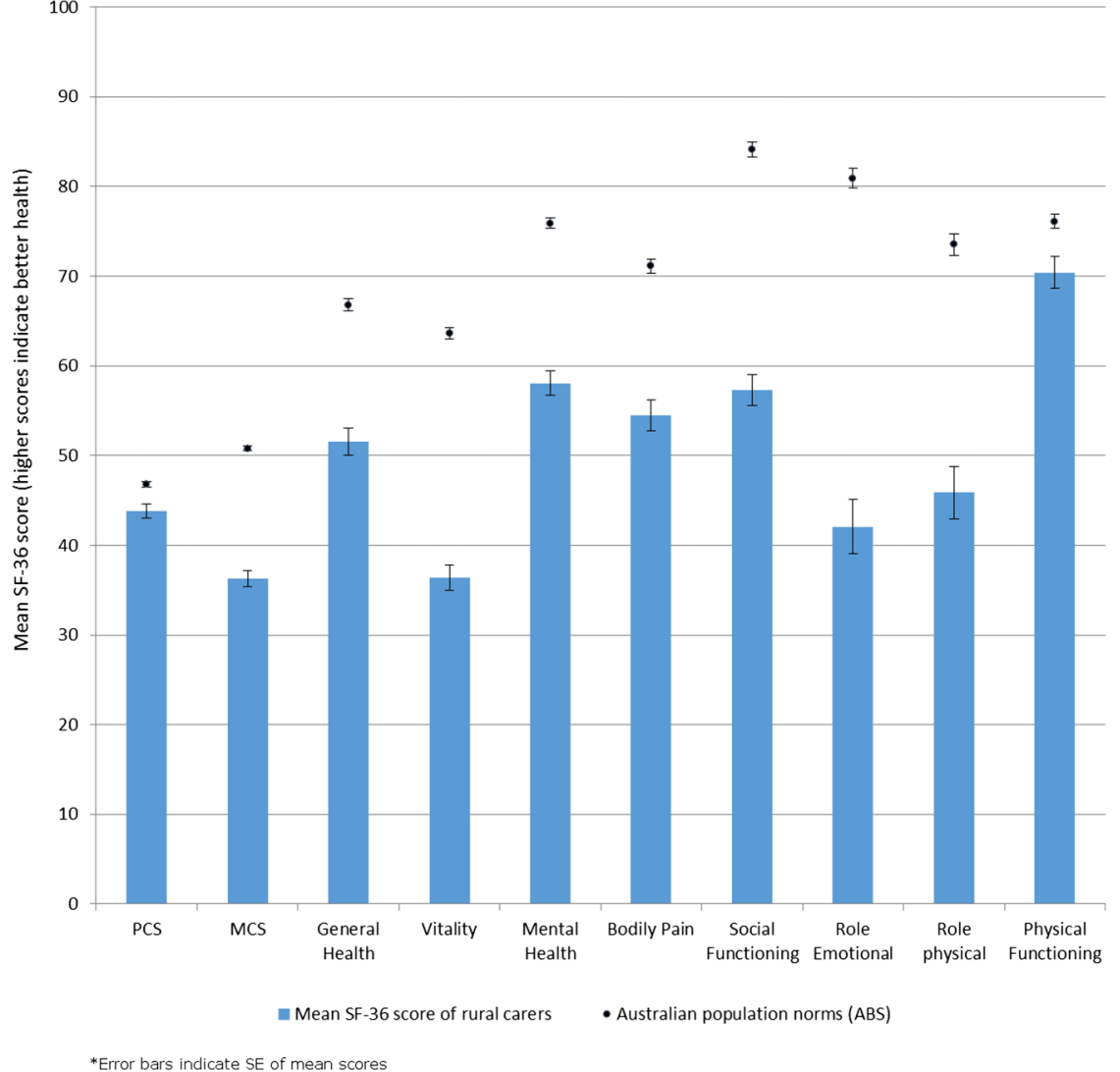

*Error bars indicate SE of mean score

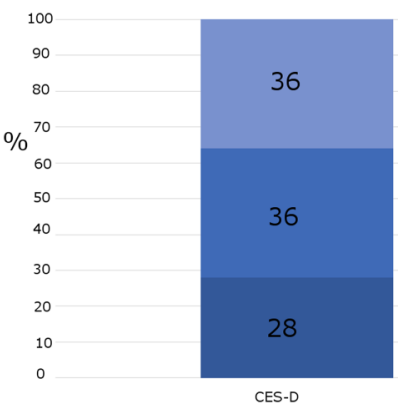

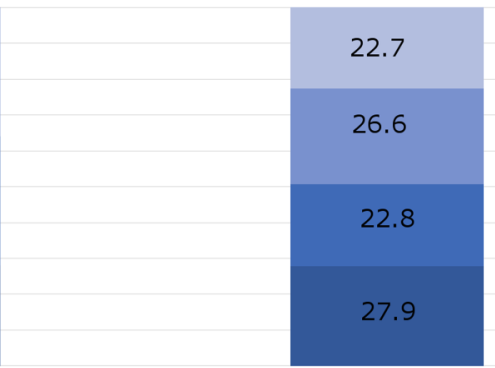

K10 functioning and physical functioning domains. Additionally, there were also significant age associations with scores on the CES-D and the K-10 Scales as shown in table 2.

Age-related differences on the physical functioning domain of the SF-36 followed population norms of declining scores with age and could be considered to be a normal function of ageing. Age-related scores for the vitality domain within the current study show a trend towards increasing vitality scores with older age. Furthermore, there was no significant difference between the PCS values of younger carers (40 years and under) compared to carers who were 61 years and above. sures of PCS and MCS as well as the general health, vitality, mental health, role emotional, role physical social 


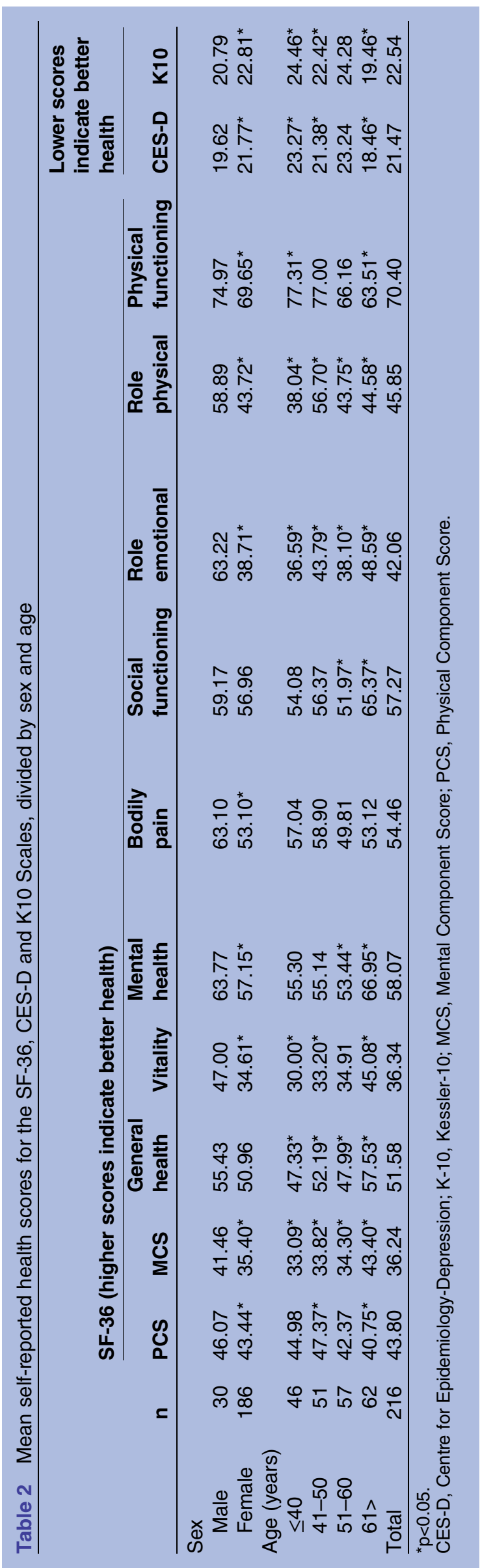

There are also several age-related associations within measures of mental health. Scores on the SF-36 MCS, mental health and social functioning domains generally increased with age indicating better aspects of mental health in older carers compared to younger age groups. Scores on the CES-D and K10 measures also indicated better mental health for older carers compared to their younger counterparts. These results, along with the findings from the SF-36 domains, can be interpreted as indicating a relatively larger mental health burden on young carers compared to older carer age groups.

Several other associations were investigated, and it was found that there were no significant differences in the mean self-reported health scores of primary versus nonprimary carers across any of the eight domains or two component scores of the SF-36, or total scores on CES-D or K-10. In addition, there were no significant differences in the mean self-reported health scores of carers from any of the relationship categories of caring for a parent/child/spouse/other. There were no significant differences in any of the mean health scores of carers for SF-36 categorised by the condition that they were caring for (physical, cognitive, mental health or multiple conditions). However, there were significantly lower scores for CES-D and K-10 of carers for physical health conditions of care recipients.

When categorised by their employment status (working yes/no), it was found that carers who were working had better self-reported health on the SF-36 PCS and physical functioning domains. There were no other significant associations of working status with health on any of the other SF-36 domains or on the K-10 or CES-D. Better physical functioning among those carers who were working was interpreted as indicating that any additional stress of working in addition to caring was not impacting negatively on the physical health of carers. The only significant difference in selfreported health between members and non-members of support groups was on the physical functioning domain of the SF-36. It was found that non-members of support groups had significantly lower scores (better health) in the domain of physical functioning compared to nonmembers (see table 2).

\section{Associations between caring relationship, condition cared for and age of carer}

There was no significant association between age of carer and the type of condition being cared for. There was however a significant relationship between the condition being cared for and the relationship between carer and recipient of care. People caring for a parent were most likely to report caring for a physical condition (66.7\%) compared to caring for a mental health or cognitive condition or multiple conditions. People caring for a child were most likely to report caring for a cognitive condition $(33 \%)$ or for multiple conditions $(33 \%)$. Although only $16.5 \%$ of people caring for a child reported caring for a mental health condition, this was 
the most common caring relationship in the mental health category. There was also a significant association between the age of the carer and the relationship with the care recipient. This would logically be related to the care of elderly parents or spouses, with $50 \%$ of carers in the 51-60 years age group reporting that they were caring for a parent and $55 \%$ of carers in the $61+$ years age group reporting that they were caring for a spouse.

In table 3, we outline the results of the multivariable regression analyses for each of the four outcome variables. As mentioned in the 'Methods' section, we used multiple linear regression for regression models for the two summary component scores of SF-36, namely PCS and MCS; and binary logistic regression for CES-D and $\mathrm{K}-10$. After adjusting for an explanatory variable such as gender, work status, condition cared for and membership of carer support group, the only statistically significant category for PCS was the 51-60 years age group. On the other hand, for MCS all age categories were significantly associated with poor mental health when compared to the $60+$ age group. The results for CES-D were somewhat anomalous, with three times higher odds of high CES-D scores indicating depression, though the results were marginally significant $(\mathrm{p}=0.06)$. For $\mathrm{K}-10$, there were three times higher odds of poor mental health across all age groups and results for all the age categories were statistically significant (see table 3 ).

\section{DISCUSSION}

The need to support another individual in basic activities of daily living is becoming an increasingly important issue in Australia. It is estimated that one in eight people provides informal and unpaid support to another person, and the demand for this type of care is predicted to increase substantially over the next 10 years. ${ }^{11}$ It is known that the provision of informal care is associated with health decline in several areas, including psychological distress, ${ }^{6}$ physical manifestations $^{7}$ and general quality of life. ${ }^{8}$ It is further understood that there is a higher proportion of informal carers in rural localities compared to metropolitan locations, ${ }^{11}$ and it is recognised that healthcare issues are potentially worse in country areas where access to healthcare services may be compromised. ${ }^{28}$ The SF-36 Scale has been used in some Australian longitudinal studies such as the Household Income and Labour Dynamics Australia (HILDA), using a panel survey design, ${ }^{44}$ and the Women's Health Australia (WHA) study. ${ }^{45}$ However, the HILDA survey does not have a specific focus on information about caregiving issues and its impact on health, whereas the WHA is limited to women participants in specific age cohorts.

The aim of the present study was to establish an initial self-reported health profile, physical and mental health, of rurally based informal carers with a secondary objective of comparing the results to Australian normative data. It was found that the self-reported physical and

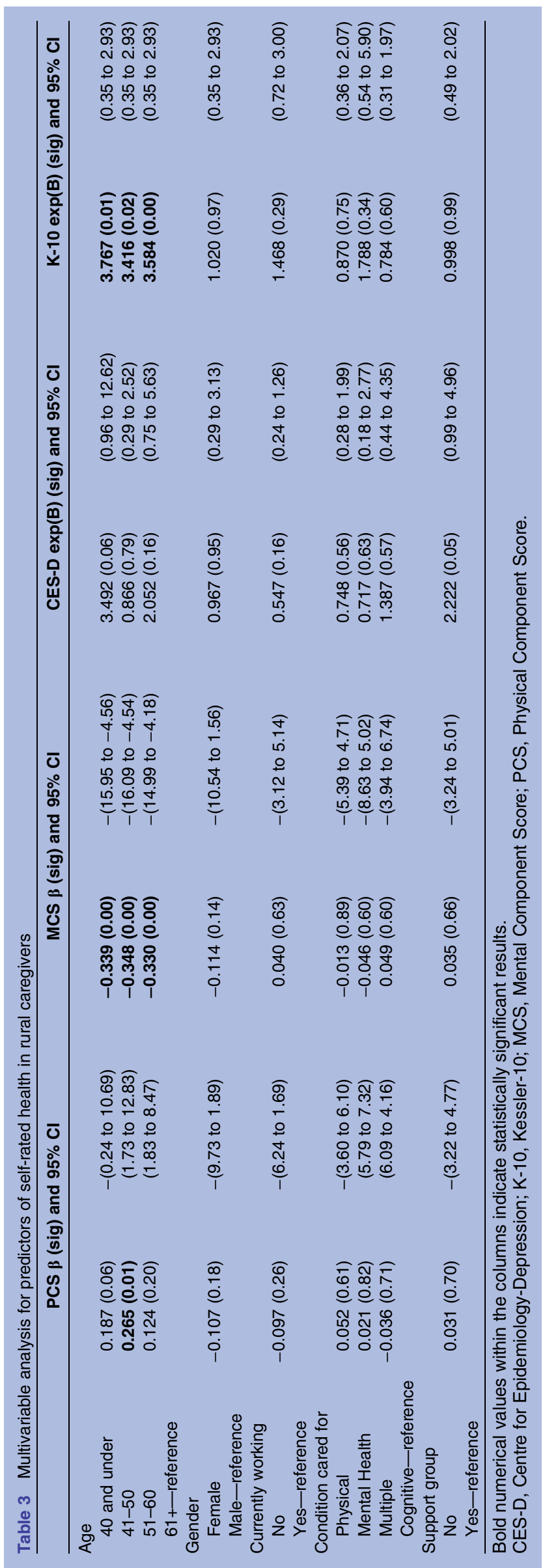


psychological health of carers within this study was significantly worse than the Australian population norms across all eight domains and the two summary component scores of the SF-36. Additionally, almost half of the carers reported high or very high level of psychological distress on the K-10 Scale. This is indicative of a major health burden related to the role of informal caregiving. It is acknowledged that, as an initial study, not all key demographic data were able to be captured. In particular, it is recommended that future research specifically examine two additional factors, the length of time the individual had been providing care and whether the care recipient was receiving palliative or end-of-life care, as it is acknowledged that these issues may have a further impact on carers' health.

The current study had a majority of female participants, which is in line with the gendered nature of informal caregiving in Australia. The nationally based Disability, Ageing and Carers Survey ${ }^{46}$ reports that, within Australia, females represent $70 \%$ of primary carers and $56 \%$ of carers overall. In the current study, male carers had significantly higher levels of selfreported health than female carers in the SF-36 MCS value as well as the health domains of vitality, role emotional and bodily pain. The additional caregiving burden for female carers has been highlighted by several previous studies, and factors related to this additional burden have been identified as including the multiple caring roles of women, especially related to childrearing and household duties, ${ }^{47}$ spending more time with care recipients than male caregivers ${ }^{48}$ and seeking less social and tangible support than male carers. ${ }^{49}$ The current study provides additional data to indicate a trend towards a higher health-related burden for female carers compared to male carers.

There were also age-related differences in selfreported health of carers that went against expected trends. For example, the Physical Health Component (PCS) score of younger carers (40 years and under) was not significantly different to the PCS score of carers aged 61 years and over. This is contrary to the expected decline in self-reported health with increasing age, as evidenced in the Australian normative SF-36 data. Furthermore, scores in the SF-36 domain of vitality showed a significant increase with age. This is in contrast to the age-related trend in the population norm data, which showed a decline in vitality scores in older age groups. ${ }^{37}$ These contrary findings to the population norm trend could be interpreted as indicating that the burden of caring on physical health may be more prominent in young carers compared to older carers within the current study. In the normative data from the Australian population, there is a decline in social functioning with age, which also is contrary to the data from the current study.

These trends indicate a greater health burden for younger carers compared to older carers. The additional care burden experienced by younger carers may be attributed to the fact that carers in the under 40 age group may be raising a family in addition to meeting the responsibilities of a caring role. The life stage prior to 60 years of age represents the key time for engagement in career and employment, and being a carer in this age group may contribute significant additional life stress, as dual responsibilities of work and caring roles compete for time and priority. ${ }^{17}$ Decisions to work in a part-time capacity, or to withdraw from the paid workforce due to caring commitments, carry significant financial consequences in the short term and for future employment prospects and long-term financial security. The perceived financial cost of caregiving has been found to be a significant contributor to the overall caregiving burden of informal carers,${ }^{50}$ and this perceived financial loss may be greater for younger carers compared to older carers.

Normative data indicate an increase in the MCS and mental health scores of older participants, so the current data are in line with this trend. However, the score increases within the current study are larger than those seen in the normative data set, with an increase of over 11 points on the mental health scores in the older group compared to the youngest group of carers, whereas a comparative age difference in the normative data is associated with only a 0.5 point difference in mean mental health scores. Considerably higher psychological distress scores were found in the present study which were over four times the rates expected in the general Australian population (12.6\%) with only $28 \%$ of study carers indicating low levels of psychological distress compared to $64.3 \%$ of the general population. ${ }^{37}$

The high level of psychological distress and depressive symptoms among the carers in this sample is a worrying result, with over $70 \%$ of the carers indicating depressive symptoms and almost half of the sample reporting high or very high levels of psychological distress. This alarmingly high rate of mental distress highlights an urgent need for additional support for informal carers in rural areas of NSW. A meta-analysis of factors contributing to physical health of informal caregivers ${ }^{49}$ reported that depressive symptoms of caregivers had a higher association with declines in physical health than objective care-related stressors such as hours of care provision, behavioural problems of the care recipient and medical condition of the care recipient and access to support. It then follows that measures to increase the mental health and well-being of carers will also have flow on benefits for physical well-being.

\section{Limitations}

This study had a number of limitations that need to be considered. Any community-based study seeking participants study cannot eliminate selection bias as a risk. It is considered unlikely that carers with more significant burdens of ill-health would have opted to participate in the survey. As such, the results reported for the present study may be an under-representation of mental health 
issues given the significant challenges of caregiving for rural carers and with the potential of more distressed carers not being able to find the time and/or energy to participate in the study. As noted in the 'Discussion' section, there were two factors that were not captured in the data, the length of time the individual had been providing care and the care recipients' stage of illness. It is acknowledged that these issues may have consequences for carers' health, but it is not believed that the failure to gain these data invalidates the reported outcomes. Nonetheless, it is recommended that future research consider stratifying the results by these variables to measure if there is any impact.

Measurement bias is largely eliminated by using validated scales for outcome variables such as SF-36, CES-D and K-10. However, for any self-report scales it is acknowledged that recall bias may be operative in the reporting of health conditions, such as bodily pain and physical functioning. It is noted that the Australian population normative data for the SF-36, collected in 1995, may now be dated given changes in population health over the past 20 years. However, these data were the most recent, of suitable format, that could be found. More recent Australian health surveys (eg, 45 and Up Study ${ }^{51}$ ) have included some aspects of the SF-36 in their reporting, but there was no alternative source found that reported gender specific, age-based, Australian population means for the full eight domains and two component scores of the SF-36. The limited number of male respondents in the current study meant that sex-based comparisons need to be viewed with caution. It is recommended that in future carer studies active recruitment of male participants may be needed in order to gain a sex ratio that is in line with the Australian overall ratio of male-to-female carers.

\section{CONCLUSIONS}

Despite the noted limitations, the current study has contributed to the health-related data of Australian carers by providing a profile of self-reported health for carers in non-metropolitan areas of NSW, Australia. These findings indicate the poor health profile of this group relative to the general Australian population, especially in terms of high levels of psychological distress, and highlight the need for additional support for rural carers in order to ease the accumulated mental and physical health burdens of this group. The need for access to specifically targeted psychological interventions for individuals at risk is clear. Recommendations for future research to build on these findings include examination of the previously identified factors of length of time the individual had been providing care and the care recipients' stage of illness, as well as undertaking a comprehensive study that comparatively examines carers residing in a variety of rural settings against metropolitan-based peers in order to recognise difference and disparity in health status.
Acknowledgements The authors thank the CReDITSS team at CCEB, Hunter Medical Research Institute, University of Newcastle, for their statistical advice, data management of SF-36 data domains and imputing of missing values across SF-36, CES-D and K-10 validated scales as per established algorithms. Further statistical advice provided by Methuen Morgan, Harley Macnamara and Stuart Fisher from the University of New England is also gratefully acknowledged.

Contributors $\mathrm{RH}$ was the chief investigator of the study. $\mathrm{RH}$ and SW designed the study. RH, SW and PR were responsible for data collection, while all authors were responsible for data analysis and interpretation. All the authors helped to draft the manuscript and approved the final version.

Funding The authors acknowledge the support provided by University of New England through the University Research Seed (URS) grant scheme. The authors further acknowledge the contribution made by the Collaborative Research Network on Mental Health and Well-being in Rural Communities, supported by the Department of Industry, Innovation, Science, Research and Tertiary Education, Commonwealth Government of Australia.

Competing interests None declared.

Patient consent Obtained.

Ethics approval University of New England Human Research Ethics Committee.

Provenance and peer review Not commissioned; externally peer reviewed.

Data sharing statement No additional data are available.

Open Access This is an Open Access article distributed in accordance with the Creative Commons Attribution Non Commercial (CC BY-NC 4.0) license, which permits others to distribute, remix, adapt, build upon this work noncommercially, and license their derivative works on different terms, provided the original work is properly cited and the use is non-commercial. See: http:// creativecommons.org/licenses/by-nc/4.0/

\section{REFERENCES}

1. Commonwealth Government of Australia. National Disability Insurance Scheme. 2015. http://www.ndis.gov.au/ (accessed May 2016).

2. Department of Social Services. My Aged Care: Help at Home. 2015. http://www.myagedcare.gov.au/help-home (accessed May 2016).

3. Hammond T, Weinberg MK, Cummins RA. The dyadic interaction of relationships and disability type on informal carer subjective well-being. Qual Life Res 2014;23:1535-42.

4. Torbica A, Calciolari S, Fattore G. Does informal care impact utilization of healthcare services? Evidence from a longitudinal study of stroke patients. Soc Sci Med 2015;124:29-38.

5. Girgis A, Lambert S, Johnson C, et al. Physical, psychosocial, relationship, and economic burden of caring for people with cancer: a review. J Oncol Pract 2013;9:197-202.

6. Hirst M. Carer distress: a prospective, population-based study. Soc Sci Med 2005;61:697-708.

7. Stenberg U, Ruland CM, Miaskowski C. Review of the literature on the effects of caring for a patient with cancer. Psychooncology 2010;19:1013-25.

8. Payne S, Smith P, Dean S. Identifying the concerns of informal carers in palliative care. Palliat Med 1999;13:37-44.

9. Hassink WH, Van den Berg B. Time-bound opportunity costs of informal care: consequences for access to professional care, caregiver support, and labour supply estimates. Soc Sci Med 2011;73:1508-16.

10. Heitmueller A. The chicken or the egg? Endogeneity in labour market participation of informal carers in England. $J$ Health Econ 2007;26:536-59.

11. Deloitte Access Economics. The economic value of informal care in Australia in 2015. 2015. http://www.carersaustralia.com.au/ storage/Access\%20Economics\%20Report.pdf

12. Australian Bureau of Statistics (ABS). 4436.0-Caring in the Community, Australia, 2012. 2014. http://www.abs.gov.au/ausstats/ abs@.nst/mf/4436.0 (accessed May 2016).

13. Cameron J, Rhodes KL, Ski CF, et al. Carers' views on patient self-care in chronic heart failure. J Clin Nurs 2016;25:144-52.

14. Anderson $\mathrm{NH}$, Gluyas $\mathrm{C}$, Mathers $\mathrm{S}$, et al. "A monster that lives in our lives": experiences of caregivers of people with motor neuron disease and identifying avenues for support. BMJ Support Palliat 
Care 2016; Published 13 May, doi:http://dx.doi.org/10.1017/ S1478951516000341

15. Aoun SM, Deas K, Kristjanson LJ, et al. Identifying and addressing the support needs of family caregivers of people with motor neurone disease using the Carer Support Needs Assessment Tool. Palliat Support Care 2016:1-12.

16. Brodaty $\mathrm{H}$, Woodward $\mathrm{M}$, Boundy $\mathrm{K}$, et al. Prevalence and predictors of burden in caregivers of people with dementia. Am J Geriatr Psychiatry 2014;22:756-65.

17. Singh P, Hussain R, Khan A, et al. Carers' perspectives on sustainability of informal care for people with dementia. SAGE Open 2015;5:1-11.

18. Zhang MW, Chan S, Wynne O, et al. Conceptualization of an evidence-based smartphone innovation for caregivers and persons living with dementia. Technol Health Care 2016(Preprint):1-5. http://dx.doi.org/10.3233/THC-161165

19. Lambert S, Pallant JF, Girgis A. Rasch analysis of the Hospital Anxiety and Depression Scale among caregivers of cancer survivors: implications for its use in psycho-oncology. Psychooncology 2011;20:919-25.

20. Zapart S, Kenny P, Hall J, et al. Home-based palliative care in Sydney, Australia: the carer's perspective on the provision of informal care. Health Soc Care Community 2007;15:97-107.

21. McNamara B, Rosenwax L. Which carers of family members at the end of life need more support from health services and why? Soc Sci Med 2010;70:1035-41.

22. Dawson S, Gerace A, Muir-Cochrane E, et al. Carers' experiences of accessing and navigating mental health care for older people in a rural area in Australia. Aging Ment Health 2015:1-8.

23. Hussain R, Maple M, Hunter SV, et al. The Fly-in Fly-out and Drive-in Drive-out model of health care service provision for rural and remote Australia: benefits and disadvantages. Rural Remote Health 2015;15:3068

24. Wark S, Hussain R, Edwards H. Ageing with an intellectual disability: support issues in rural localities. Proceedings of the 12th National Rural Health Conference National Rural Health Alliance; Adelaide, Australia, 2013.

25. Edwards B, Gray M, Baxter J, et al. The tyranny of distance? Carers in regional and remote areas of Australia. Canberra: Australian Institute of Family Studies, 2009.

26. Wark S, Hussain R, Edwards $H$. Rural and remote area service provision for people aging with intellectual disability. J Policy Pract Intellect Disabil 2013;10:62-70.

27. Dew A, Veitch C, Lincoln M, et al. The need for new models for delivery of therapy intervention to people with a disability in rural and remote areas of Australia. $J$ Intellect Dev Disabil 2012;37:50-3.

28. Wark S, Hussain R, Edwards H. Impediments to community-based care for people ageing with intellectual disability in rural New South Wales. Health Soc Care Community 2014;22:623-33.

29. Vecchio N. The use of support systems by informal caregivers: an Australian experience. Aust J Prim Health 2008;14:27-34.

30. lacono T, Humphreys J, Davis $\mathrm{R}$, et al. Health care service provision for country people with developmental disability: an Australian perspective. Res Dev Disabil 2004;25:265-84.

31. Ervin K, Pallant J, Reid C. Caregiver distress in dementia in rural Victoria. Australas J Ageing 2015;34:235-40.

32. Australian Bureau of Statistics (ABS). 3235.0-Population by Age and Sex, Regions of Australia, 2013, Main Features. 2013. http://www.abs. gov.au/Ausstats/abs@.nsf/mf/3235.0 (accessed May 2016).

33. Australian Bureau of Statistics (ABS). 3235.0-Population by Age and Sex, Regions of Australia. 2013. http://www.abs.gov.au/ Ausstats/abs@.nsf/Latestproducts/3235.0Main\%20Features22013? opendocument\&tabname $=$ Summary $\&$ prodno $=3235.0$ \&issue $=$ 2013\&num=\&view = (accessed Jul 2015).
34. Australian Bureau of Statistics (ABS). Remoteness Structure. Secondary Remoteness Structure, 10 June 2014. http://www.abs. gov.au/websitedbs/d3310114.nsf/home/remoteness+structure (accessed Jul 2015).

35. Ware JE, Kosinski M, Dewey JE, et al. SF-36 health survey: manual and interpretation guide. Lincoln (RI): Quality Metric, 2000.

36. Downey RG, King CV. Missing data in Likert ratings: a comparison of replacement methods. J Gen Psychol 1998;125: 175-91.

37. Australian Bureau of Statistics (ABS). 4399.0-National Health Survey: SF-36 Population Norms, Australia, 1995. 3 July 2008. 1997. http://www.abs.gov.au/AUSSTATS/abs@.nsf/DetailsPage/ 4399.01995 (accessed Sep 2015)

38. Radloff LS. The CES-D scale a self-report depression scale for research in the general population. Appl Psychol Meas 1977;1:385-401.

39. Smarr KL, Keefer AL. Measures of depression and depressive symptoms: Beck Depression Inventory-II (BDI-II), Center for Epidemiologic Studies Depression Scale (CES-D), Geriatric Depression Scale (GDS), Hospital Anxiety and Depression Scale (HADS), and Patient Health Questionnaire-9 (PHQ-9). Arthritis Care Res (Hoboken) 2011;63(Suppl 11):S454-66.

40. McDowell I. Measuring health: a guide to rating scales and questionnaires. Oxford University Press, 2006.

41. Crawford J, Cayley C, Lovibond PF, et al. Percentile norms and accompanying interval estimates from an Australian general adult population sample for self-report mood scales (BAI, BDI, CRSD, CES-D, DASS, DASS-21, STAI-X, STAI-Y, SRDS, and SRAS). Austr Psychol 2011;46:3-14.

42. Australian Bureau of Statistics (ABS). 4817.0.55.001-Information Paper: Use of the Kessler Psychological Distress Scale in ABS Health Surveys, Australia, 2001. 21 May 2003. http://www.abs.gov. au/ausstats/abs@.nsf/papersbyReleaseDate/4D5BD324FE8B415F CA2579D500161D57?OpenDocument (accessed May 2016).

43. Field A. Discovering statistics using SPSS. London: Sage, 2009.

44. Butterworth P, Crosier T. The validity of the SF-36 in an Australian National Household Survey: demonstrating the applicability of the Household Income and Labour Dynamics in Australia (HILDA) Survey to examination of health inequalities. BMC Public Health 2004:4:1-11.

45. Lee C, Gramotnev H. Transitions into and out of caregiving: Health and social characteristics of mid-age Australian women. Psychol Health 2007;22:193-209. http://dx.doi.org/10.1080/147563606 00682202

46. Australian Bureau of Statistics (ABS). 4430.0—Disability, Ageing and Carers, Australia: Summary of Findings, 2012. 13 November 2013. http://www.abs.gov.au/ausstats/abs@.nsf/Lookup/ 5968BE956901DD79CA257D57001F4D89?opendocument (accessed May 2016)

47. Wallace Williams S, Dilworth-Anderson P, Goodwin PY. Caregiver role strain: the contribution of multiple roles and available resources in African-American women. Aging Ment Health 2003;7:103-12.

48. Chiou CJ, Chen IP, Wang HH. The health status of family caregivers in Taiwan: an analysis of gender differences. Int $J$ Geriatr Psychiatry 2005;20:821-6.

49. Pinquart M, Sörensen S. Correlates of physical health of informal caregivers: a meta-analysis. J Gerontol B Psychol Sci Soc Sci 2007;62:P126-37.

50. Lai DW. Effect of financial costs on caregiving burden of family caregivers of older adults. SAGE Open 2012;2:1-14.

51. Sax Institute. 45 and Up Study. Secondary 45 and Up Study 2015. https://www.saxinstitute.org.au/our-work/45-up-study/ (accessed May 2016). 